\title{
Ebstein repair: How I do it
}

\author{
Joseph A. Dearani, MD
}

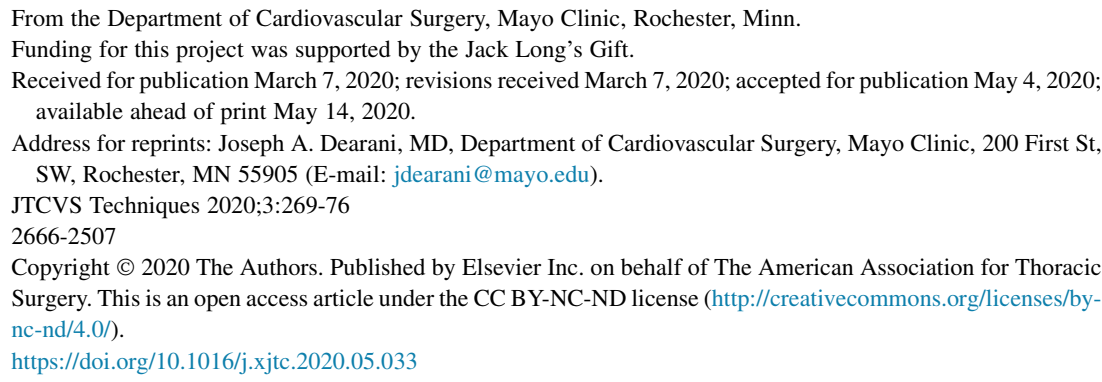

The most contemporary repair technique that provides a near-anatomic reconstruction was described by da Silva and colleagues. ${ }^{1}$ More recent outcomes in larger numbers of patients undergoing cone repair have been reported, ${ }^{2,3}$ and modifications to the original cone repair including re-repair techniques have also been described. ${ }^{4}$ In contrast to previous techniques that focused on an anterior leaflet monocusp method, the cone repair results in $360^{\circ}$ of tricuspid leaflet tissue surrounding the right atrioventricular junction. This allows leaflet-to-leaflet tissue coaptation, similar to what occurs with normal tricuspid valve anatomy. In addition, the reconstructed tricuspid valve is reattached at the true tricuspid valve annulus so the valve hinge point is now in a nearly normal anatomical location. Atrialized right ventricle (RV) (typically the inferior wall) is plicated to eliminate areas of RV dyskinesis, and redundant right atrium is excised. The cone reconstruction restores the appearance of near-normal tricuspid valve anatomy (Figure 1) and function more than previously described techniques that revolved mostly around a monocusp repair with coaptation of the anterior leaflet with the ventricular septum or diminutive septal leaflet.

We have gained substantial surgical experience with Ebstein anomaly since 1972 (overall >1400; Cone repair 400). The purpose of this review is to share the lessons we have learned with tricuspid valve repair in the setting of Ebstein anomaly, and many of these principles can be applied to other congenital and acquired pathologies of the tricuspid valve.

\section{OPERATIVE TECHNIQUE}

The operation is performed via median sternotomy; mini sternotomy or limited right thoracotomy has been applied selectively in young adults. Intraoperative transesophageal echocardiogram is used routinely. Standard cardiopulmonary bypass techniques with aortic and bicaval cannulation is used. Normothermia or mild systemic hypothermia $\left(34^{\circ} \mathrm{C}\right)$ and cardioplegic arrest with aortic crossclamping and cold blood antegrade cardioplegia (del Nido $30 \mathrm{cc} / \mathrm{kg}$ )

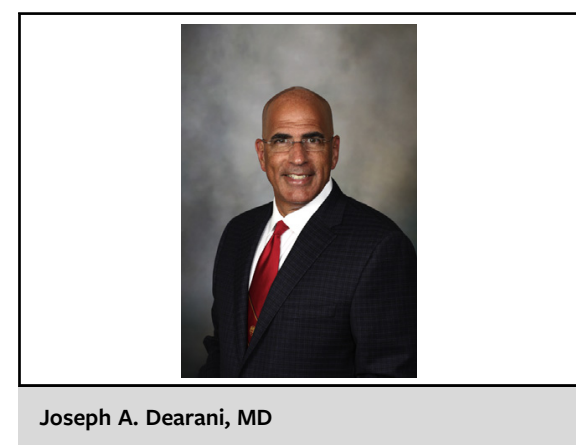

CENTRAL MESSAGE

Cone repair for Ebstein anomaly results in circumferential leaflet tissue surrounding the atrioventricular junction.

See Commentaries on pages 277 and 279.

is used. Asystolic arrest is maintained with subsequent doses $(10-15 \mathrm{cc} / \mathrm{kg})$ of antegrade blood cardioplegia given at 60-minute intervals during aortic occlusion. The main pulmonary artery is included with the aortic crossclamp, as it facilitates analysis of the tricuspid valve after repair when the RV is filled with saline via bulb syringe.

An oblique right atriotomy is performed from the right atrial appendage toward the inferior vena cava that is parallel to the atrioventricular groove. The left heart is vented with a catheter inserted across the atrial septum. Exposure of the tricuspid annulus is facilitated with stay sutures placed in the true tricuspid annulus at 10- and 2-o'clock positions. Preliminary examination of the valve is done with saline injection into the RV to determine the extent of leaflet mobility, sites of tethering, and areas of noncoaptation, and the atrialized RV is evaluated. The membranous septum and atrioventricular node are identified by a small vein (vein of D) in fatty tissue that is characteristically present.

The anterior leaflet is detached at the annulus at approximately 8-o'clock position (close to the anteroseptal commissure). The incision is extended clockwise around to the inferior annulus toward the coronary sinus; this incision ends when leaflet tissue ends and is often in the area of 2-o'clock position, where inferior leaflet tissue is diminutive or absent, or further rightward (2- to 4-o'clock positions) when inferior leaflet tissue is present. It is common for a true space to be present between the anterior leaflet and the RV in various areas consistent with adequately 


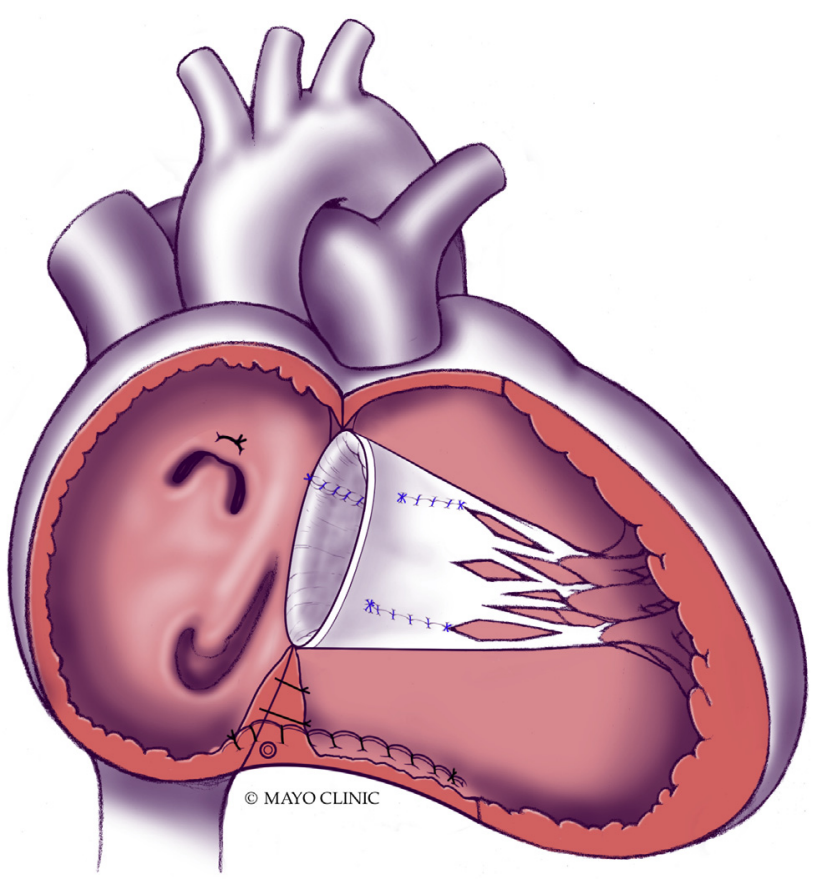

FIGURE 1. Completed Ebstein repair that includes cone reconstruction of the tricuspid valve (circumferential leaflet tissue surrounding the atrioventricular junction), plication of the atrialized right ventricle inferiorly, and right reduction atrioplasty.

delaminated leaflet. However, when the transition is met between the anterior and inferior leaflets, it is common to have failure of delamination resulting in fibrous and muscular attachments between the leaflet and RV myocardium. The dissection continues in a way that a portion of anterior leaflet and available inferior leaflet is "surgically delaminated," ie, surgically peeled off of the RV (Figure 2). The most important aspect of surgical delamination is to incise (and excise) all fibrous and muscular attachments between the body of the leaflet and the RV free wall but to maintain intact all fibrous and (occasionally muscular) attachments to the leading edge of the leaflet(s). Leaflet muscularization is sometimes noted in the anterior and inferior leaflets...this is debulked as much as possible to improve and optimize leaflet pliability. It is important to examine the leaflet anatomy from both sides. Specifically, the leading edge of the leaflet is examined to see whether there are individual chordal attachments present or whether a broad segment of the leading edge is attached directly to the myocardium - this is referred to as a "linear attachment" "; management of this abnormality is described to follow.

In Ebstein anomaly, the septal leaflet is displaced in an apical rotational fashion into the RV. The proximal annular edge of the septal leaflet is incised and mobilized off of the ventricular septum and is continued with the goal of taking down all attachments between the body of the septal leaflet and septum but preserving all attachments of the leading

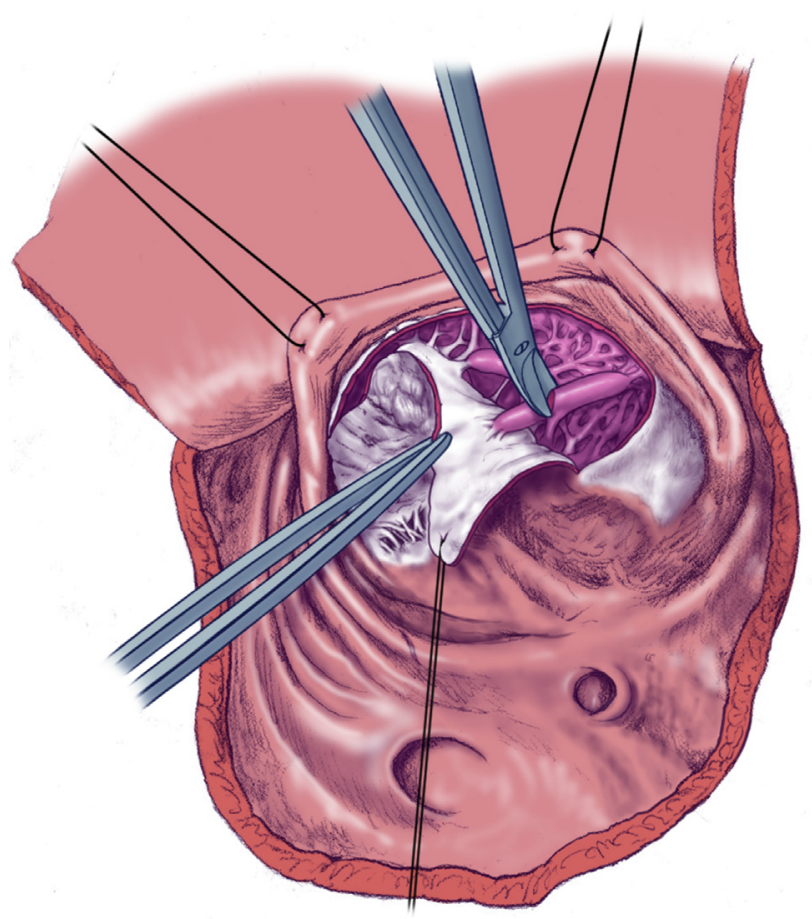

FIGURE 2. Annular anterior leaflet detachment with surgical delamination; division of muscular and fibrous attachments between the body of the leaflet(s) and anterior and inferior ventricular free walls.

edge as described previously (Figure 3). The dissection and leaflet mobilization should proceed medially until leaflet tissue ends and laterally toward the anteroseptal commissure. There can be marked variability in the leading

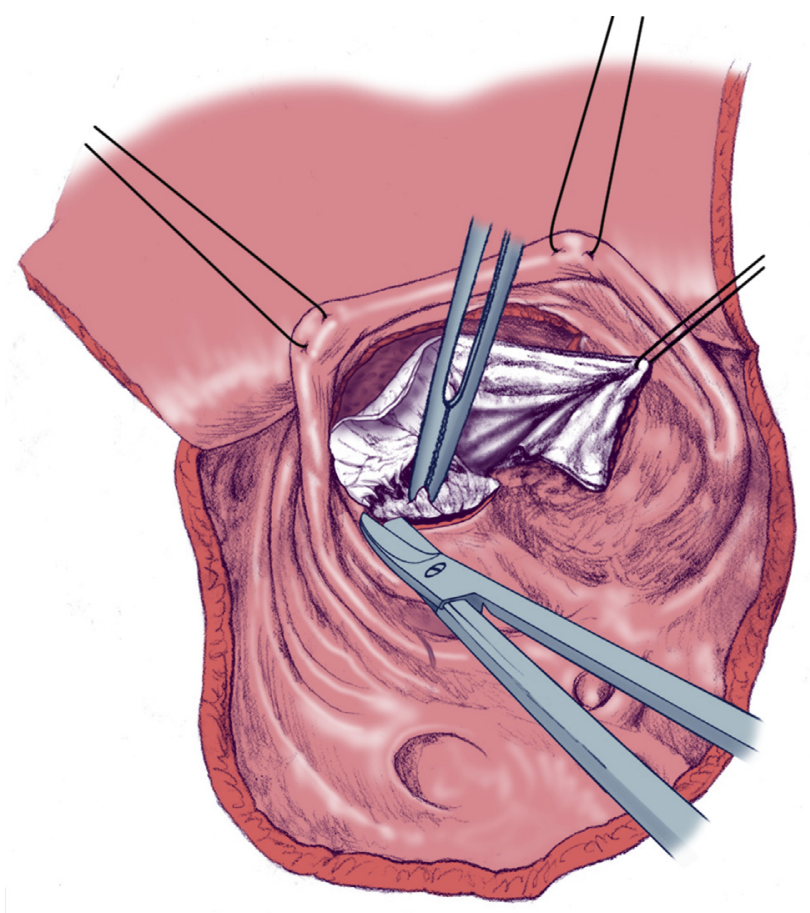

FIGURE 3. Septal leaflet mobilization. 


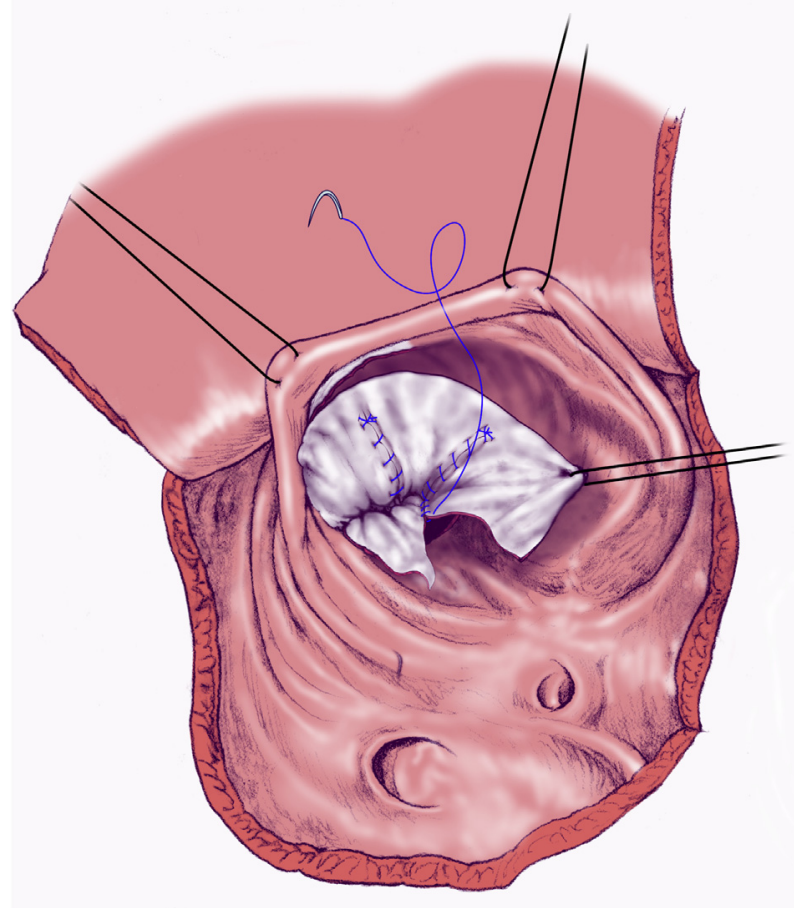

FIGURE 4. Clockwise leaflet rotation and approximation of the mobilized anterior/inferior leaflet to the proximal mobilized edge of the septal leaflet.

edge of the septal leaflet, and a linear attachment is often present.

After the anterior, inferior, and septal leaflets have been completely mobilized, the side of the cut edge of the inferior leaflet (or anterior leaflet if no inferior leaflet was present) is rotated clockwise to meet the proximal (annular) edge of the mobilized septal leaflet. The 2 edges (sides) are approximated with a fine running, locking (to avoid a purse string effect that would shorten the width or height of the leaflet reconstruction) monofilament suture creating and completing the cone of leaflet tissue (Figure 4). This results in $360^{\circ}$ of leaflet tissue that covers the new tricuspid valve orifice. In general, we try to preserve the anteroseptal commissure and do so for 2 reasons: (1) avoid septal leaflet dissection, mobilization, detachment, and reattachment in the area of the conduction tissue that could cause heart block and (2) maintain a commissure to reduce the possibility of iatrogenic tricuspid stenosis by allowing the leaflets to fall away from each other during diastole.

In situations in which the septal leaflet is absent, the anterior leaflet is completely detached at the anteroseptal commissure. Side-to-side connections are performed between all available segments of mobilized leaflet tissue, making a true "cone" with no commissures present. In this situation, it is important to ensure that the distal orifice of the cone is of adequate size to avoid tricuspid stenosis, which is more likely to occur when there is insufficient

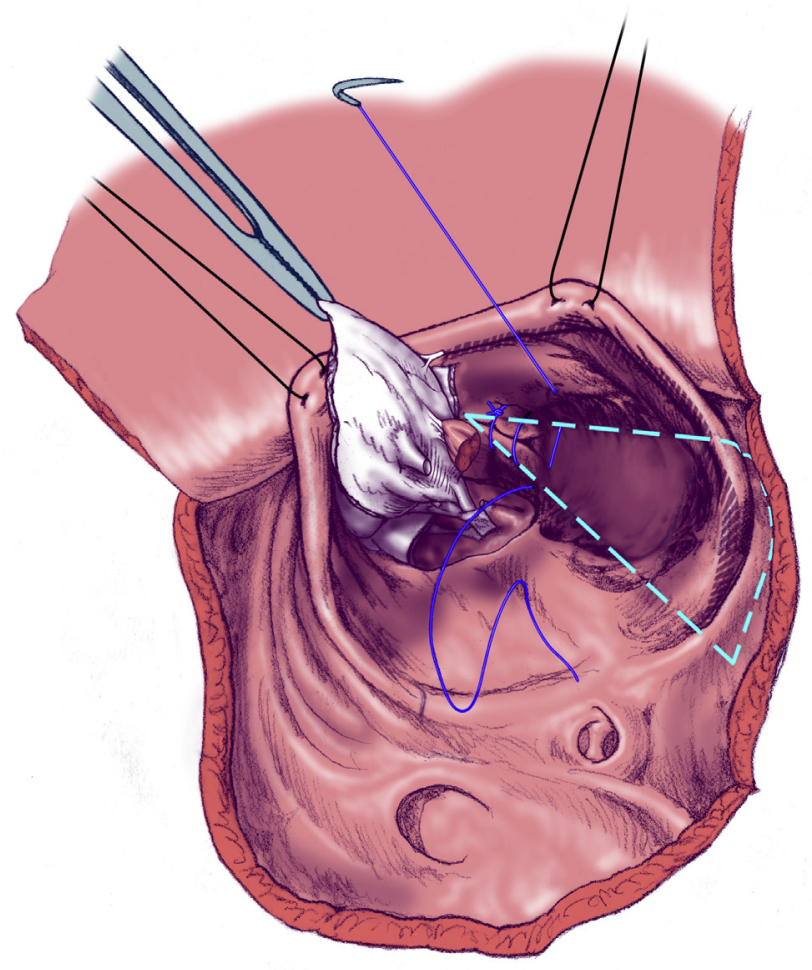

FIGURE 5. Identification of the atrialized (smooth and non-trabeculated) right ventricle inferiorly marked by the dotted triangle. The base of the triangle crosses the atrioventricular groove so the diameter of the dilated annulus is reduced in size as the plication is completed.

leaflet tissue to create a good-sized cone or when there is no commissure(s) present.

After the cone reconstruction is completed, the atrialized $\mathrm{RV}$ is examined to determine whether plication is necessary. Atrialization typically occurs along the inferior wall of the $\mathrm{RV}$; it is smooth and non-trabeculated and the line of demarcation with normal trabeculated RV muscle is clear. The technique for internal plication of the atrialized RV includes the following: monofilament suture (5-0 in children; 4-0 in adults) is used and the suture line is begun distally, ie, closest to the RV apex (Figure 5). Suture bites are confined to the endocardium to avoid a coronary compromise from sutures exiting onto the epicardial surface and distorting/ ligating acute marginal branches of the right coronary artery. It is important to periodically examine the exterior surface of the RV to ensure that inadvertent compromise of coronary branches has been avoided. The plication suture line is advanced toward the base of the heart, ie, toward the atrioventricular groove and extends across the annulus and into the right atrium. As the dotted lines of the base of the triangle are effectively approximated (Figure 5), the atrialized RV is excluded, reducing the size of the RV and the true annulus so it is closer in size to the smaller neotricuspid orifice. It should be emphasized that the plication 


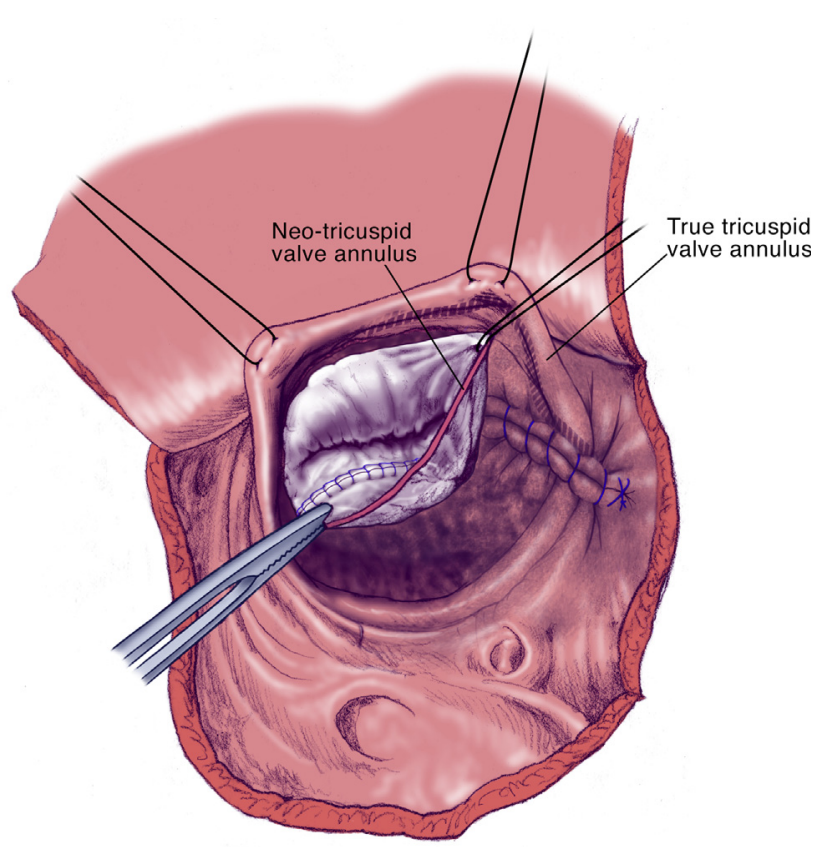

FIGURE 6. Completed plication suture line of the atrialized right ventricle. It is essential to inspect the epicardial surface of the right ventricle to confirm that there is no compromise of the right coronary artery or any of its major branches. Note persistent size discrepancy between the neotricuspid orifice and the true annulus.

suture line be confined to the atrialized segment of the inferior wall of the RV; specifically, it is important that the suture line does not inadvertently drift onto the ventricular septum (which is also smooth with minimal trabeculations), since the posterior descending coronary artery is very vulnerable to injury at this junction of the RV free wall and ventricular septum. As the suture line crosses the AV groove, it is essential to avoid deep bites that could also compromise the distal main right coronary artery (Figure 6). After the corners of the base of the triangle are approximated to eliminate the "blind pouch" into the excluded segment of inferior wall, a second suture line is completed so dehiscence is avoided.

After the RV plication is completed, the neotricuspid valve is reattached at the level of the tricuspid annulus. Since the neotricuspid valve will have an orifice that is smaller than the original dilated atrioventricular junction, additional annular plication may be necessary to match the size of the smaller neotricuspid valve orifice. Options include a single plication site at the inferior annulus when a limited annular reduction is desired. If the size discrepancy between the larger true tricuspid annulus and the smaller neotricuspid valve is substantial, smaller annular plication sutures are evenly spaced in multiple areas around the true annulus in order to avoid distortion of the right coronary artery by a large (inferior) plication in a single location.

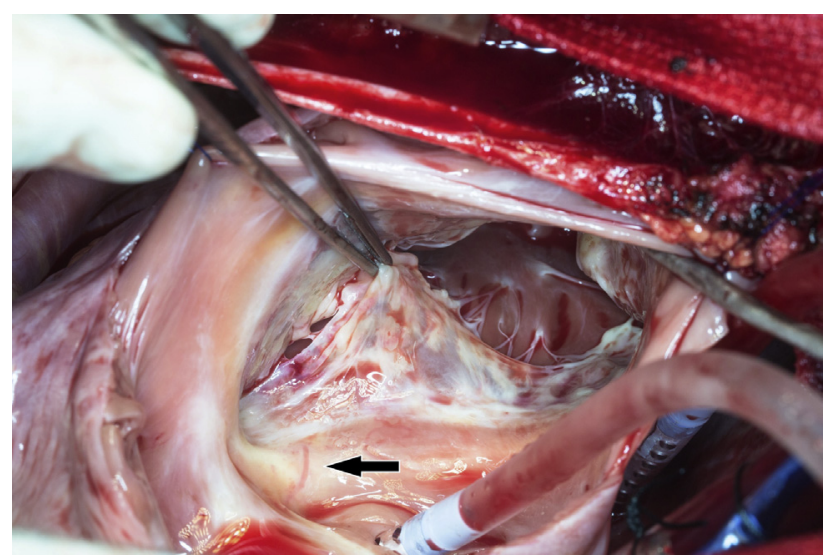

FIGURE 7. Intraoperative photo showing apical displacement of the septal leaflet. The arrow signifies the conduction tissue marked by the vein of $\mathrm{D}$ and the white membranous septum immediately to the left of the vein.

After the inferior annulus is plicated to the appropriate size to match the neotricuspid valve, the proximal cone leaflet tissue is anchored at the true annulus with fine continuous monofilament suture. To avoid heart block, the septal reattachment suture line is performed just caudal to the membranous septum and AV node that is marked by the previously described vein of D (Figure 7).

The completed cone reconstruction of the tricuspid valve is evaluated for competency with RV saline injection. Any residual fenestrations or areas of leak are repaired as needed. Natural "zones of apposition" or "clefts" are not routinely closed unless there is evidence of residual leak present in those areas. Empiric closure of these natural "clefts" can result in leaflet distortion and may cause more residual leakage; hence, it is important to wait until the completion of the repair and then examine the valve with saline injection to be sure that cleft approximation is indeed necessary.

\section{MAYO CONE MODIFICATIONS} Annular Reinforcement

The inferior tricuspid annulus is the site most vulnerable to annular dilation. This is also the location of the suture line for plication of the atrialized RV. Since there will be tension in this area with RV contraction and relaxation, annular reinforcement is done liberally. In children, the inferior annulus is supported with a short pericardial strip (1-o'clock position to the coronary sinus) (Figure 8, $A$ ) and in older children and adults, annular support is achieved with a flexible annuloplasty band $(26 \mathrm{~mm})$ from anteroseptal commissure clockwise to the coronary sinus. The site of inferior annulus plication (lateral to the band) is reinforced with a pledgeted mattress suture(s).

\section{Shallow Anterior Leaflet}

The height of the leaflet is "shallow" when there is little distance between leaflet leading edge (with its chordal 

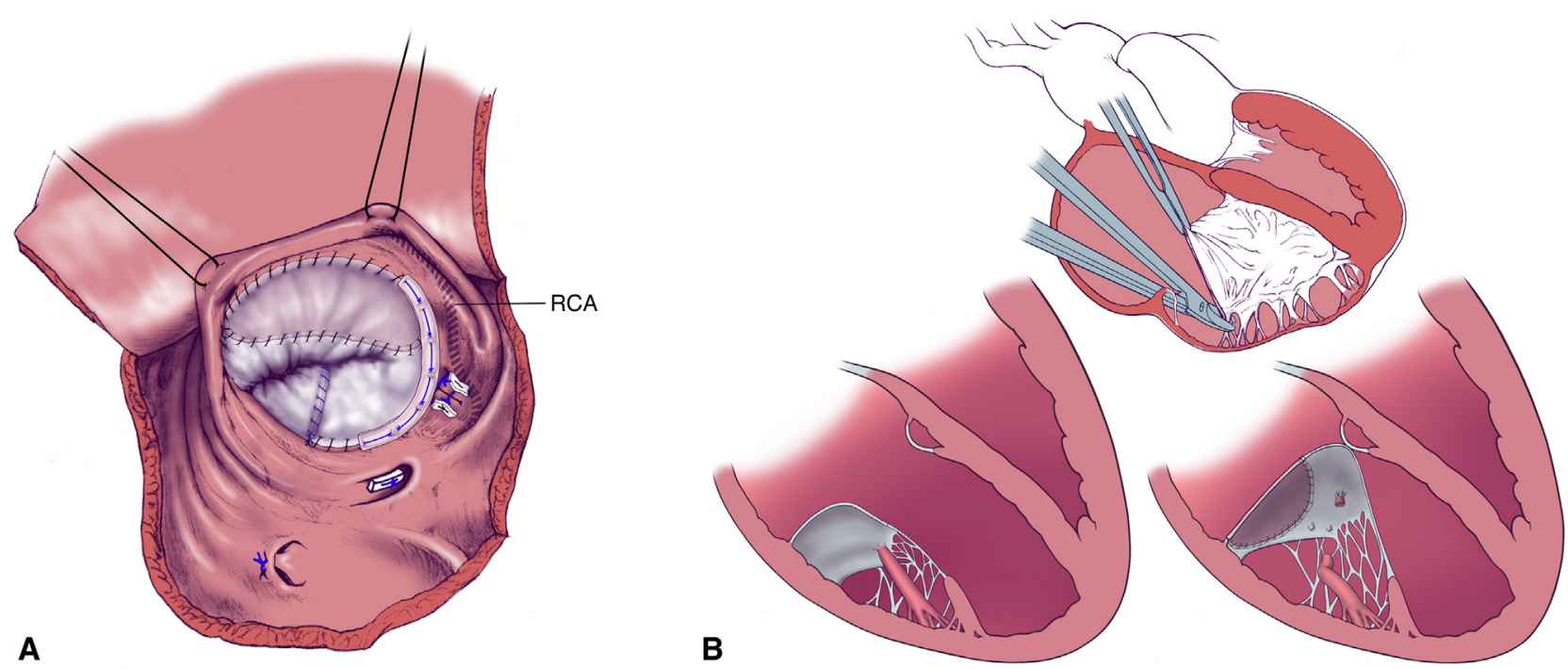

FIGURE 8. A, Completed cone repair. The anterior leaflet has been patched with an ellipse of autologous pericardium. The inferior annulus has been plicated with a pledgeted mattress suture to further reduce the size of the true annulus; note the potential vulnerability of right coronary artery kinking. Since the inferior annulus is most susceptible to dilation and is also the area under the most tension, an inferior annuloplasty band (autologous or bovine pericardium) is applied and anchored with a pledgeted sutured in the coronary sinus. Suture closure of the patent foramen ovale is also shown. B, Four-chamber schematic demonstrating surgical delamination process of muscular and fibrous attachments between the body of the leaflet and the right ventricle free wall. The anterior leaflet augmentation effectively brings the leading edge of the anterior leaflet closer to the septal leaflet and ventricular septum optimizing coaptation before additional reduction annuloplasty maneuvers. $R C A$, Right coronary artery.

support) and the annulus. In this situation, an ellipse of autologous pericardium is used to patch augment the anterior leaflet resulting in an increase in leaflet height (Figure 8, $A$ and $B$ ). This brings the leading edge of the "taller" anterior leaflet closer to the septal leaflet and ventricular septum allowing better coaptation before annular reduction maneuvers. In general, we try to avoid additional pericardial patch material.

\section{Insufficient Cone Circumference}

In this situation, autologous side-to-side connections of a limited amount of leaflet tissue results in a small cone. The circumference of the cone is increased by using a triangular piece of autologous pericardium. A side-to-side connection is done between one side of the pericardium and the proximal, mobilized edge of the septal leaflet; a second side-to-side connection is done between a side of the pericardium and the side of the mobilized side of the anterior or inferior leaflet; and the third and final side of the triangular piece of pericardium becomes the annular reattachment to the ventricular septum (Figure 9). As noted previously, we try and avoid this maneuver of additional pericardium whenever possible and try to confine the repair to all native valve tissue.

\section{Linear Attachment}

When a linear attachment (ie, leading edge tethered with absent chordae) is present (all leaflets can be affected), it is treated by making longitudinal fenestrations in the distal aspect of the mobilized leaflet - the equivalent of making natural "neochordae." (Figure 10, $A-D$ ). This is most often applied to the septal leaflet when it is just a mound of fibrous tissue on the septum with minimal or no mobility. The length of the incisions in the leaflet is typically about one quarter to one third the distance from the leaflet leading edge into the body of the leaflet.

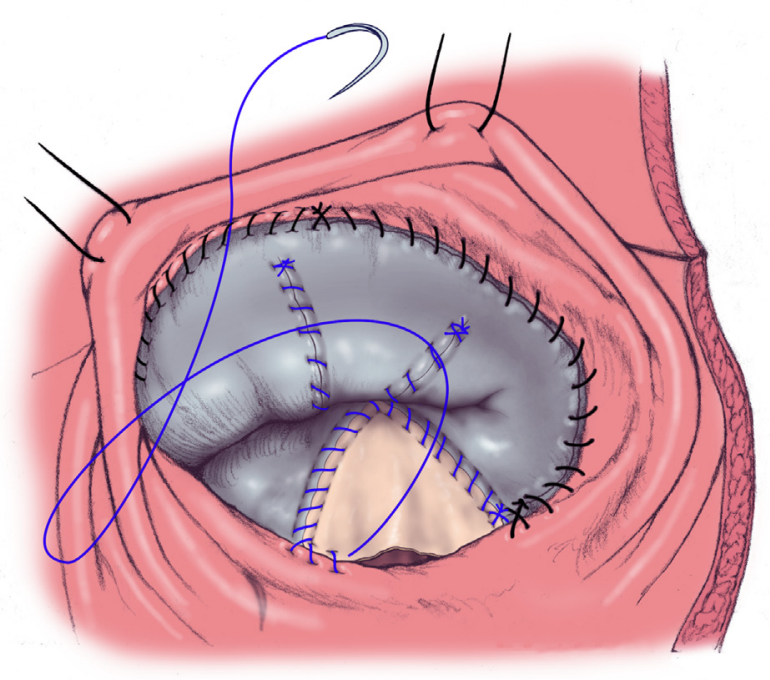

FIGURE 9. Cone circumference augmentation with a triangular-shaped piece of autologous pericardium. 


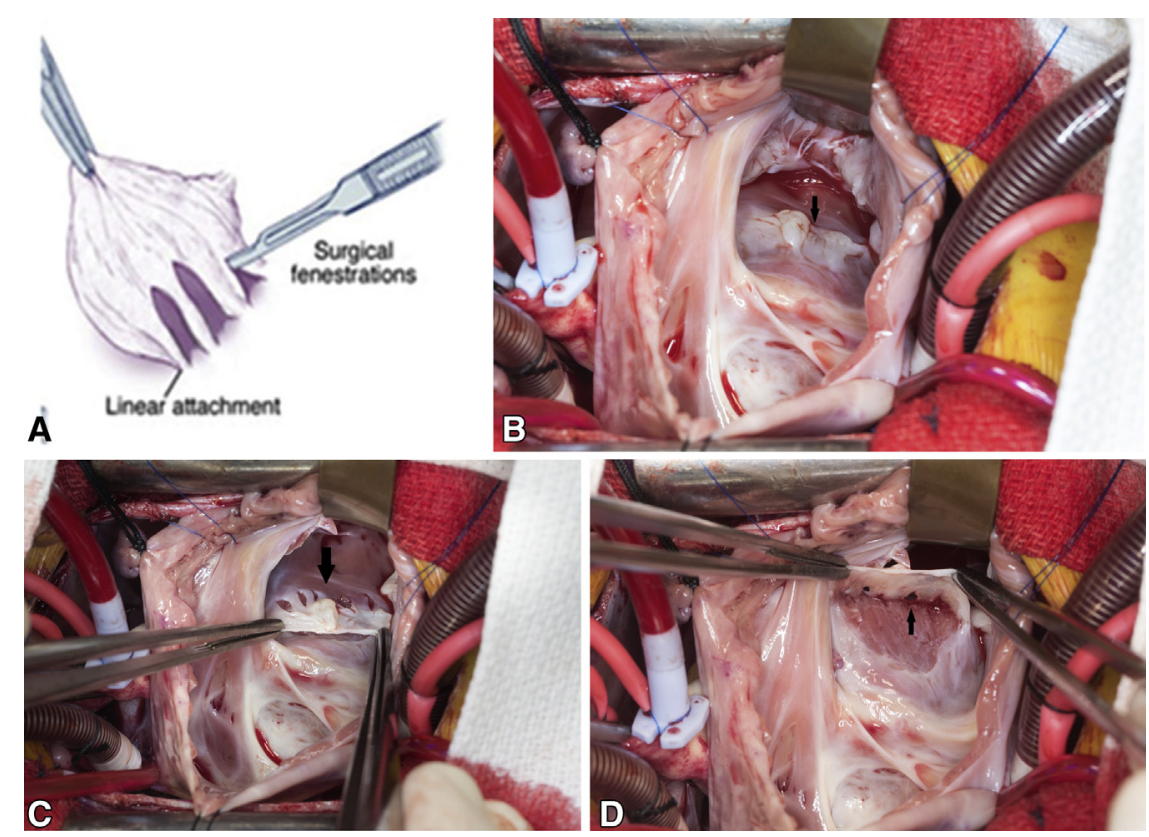

FIGURE 10. A, Creation of autologous neochordae when a linear attachment is present. In general, the leaflet cuts (fenestrations) are approximately one third the distance to the annulus. B, Intraoperative photo of a displaced, diminutive, completely immobile septal leaflet that has a linear attachment. Arrow indicates linear attachment of septal leaflet. C, The septal leaflet has been mobilized and autologous neochordae have been created (arrow). D, View of the undersurface of the mobilized septal leaflet. Autologous neochordae are in the process of being made (arrow). Further leaflet mobilization is still required and more autologous neochordae still need to be made.

\section{Paucity of Chordal Support or Leaflet Prolapse}

There are some occasions in which there is a lack of chordal support to a portion of the anterior or septal leaflet resulting in prolapse of that particular leaflet segment. When this occurs (and results in regurgitation), the use of fine Gore-Tex suture (6-0 in children; 5-0 in adults) is used to provide leaflet support. ${ }^{6}$ Importantly, the artificial chordae are anchored in trabeculations in the ventricular septum toward the apex. We generally do not anchor the artificial chord(s) in the anterior free wall papillary muscle(s), since it is difficult to gauge the proper length of the chord because of severe RV dilation in a flaccid heart. An advantage of anchoring the anterior leaflet chord into the ventricular septum is that it naturally pulls the leading edge of the leaflet toward the septum and septal leaflet, which facilitates coaptation. A pledgeted mattress suture is passed through an apical-septal trabeculation and then passed through the leading edge of the leaflet twice. The edge of the leaflet is placed in the plane of the annulus and the valve is tested by RV saline injection. The leaflet is moved inward or outward as needed until valve competence is optimized and then the Gore-Tex suture is secured. It is not uncommon for there to be some leaflet prolapse at the completion of the cone repair; if the valve is competent, then nothing is done about this. Artificial chords are only applied if leaflet prolapse results in regurgitation and, in general, they are rarely necessary.

\section{Modified Sebening Stitch}

The original Sebening stitch involved suture fixation of the major anterior RV free wall papillary muscle(s) to the ventricular septum. This approach is most applicable in the neonate that is undergoing a monocusp (or bicuspid) tricuspid repair. However, in older children and adults the original Sebening stitch is avoided because of excessive tension (long distance between papillary muscle and ventricular septum from dilated RV) and resultant dehiscence. An alternative strategy that we have described is the modified Sebening stitch, which is accomplished by approximation of the heads of the mobilized anterior and mobilized septal or inferior papillary muscles (Figure 11). This results in alignment of the leading edges of the leaflets with significantly reduced (or no) tension. Another modification of the Sebening stitch that we have used includes placement of pledgeted Gore-Tex artificial chord(s) from the ventricular septum up to the head(s) of the anterior papillary muscle(s); this is similar to Gore-Tex artificial chords described previously, but the suture is anchored into the papillary muscle head and not the leaflet leading edge. The length of the artificial chords is adjusted accordingly to pull the anterior leaflet and subvalvar apparatus closer to the septum/septal leaflet until valve competence is achieved. 


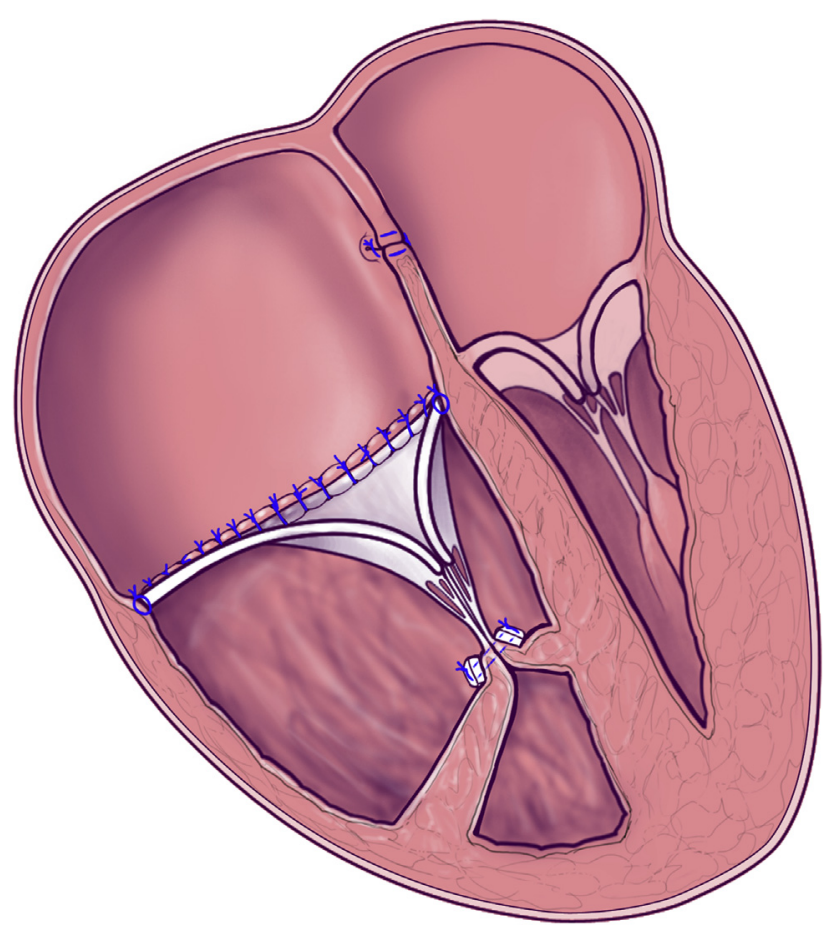

FIGURE 11. The modified Sebening stitch. This should be used rarely and when it is applied, it is important that the anterior papillary muscle be thoroughly mobilized so that it meets a corresponding septal papillary muscle (which is usually small) without tension. When this has been done correctly, there should be no dimple in the free wall of the RV after separation from bypass. A dimple in the RV free wall means tension at the approximation and should not be accepted since dehiscence will almost always occur in the early postoperative period. A concomitant annuloplasty should be applied with caution since the combination of these 2 maneuvers can result in tricuspid stenosis.

\section{THE BIDIRECTIONAL CAVOPULMONARY ANASTOMOSIS AND ATRIAL SEPTAL FENESTRATION}

In general, we prefer to avoid a bidirectional cavopulmonary anastomosis (BDCPA) and prefer to close the atrial level shunt (atrial septal defect or patent foramen ovale). There are circumstances in which there is a need to do one or the other because of the concern of low cardiac output in the postoperative period. A couple of important points to keep in mind: an atrial level shunt allows rightto-left shunting and provides some left ventricular preload. However, an atrial level shunt does not decompress the dysfunctional RV. In contrast, the BDCPA accomplishes 2 things-it provides preload to the left ventricle and it decompresses the RV by reducing systemic venous return by a factor of one third to one half depending upon the patient's age. When there is significant concern about low cardiac output from RV dysfunction, the BDCPA is a more effective strategy.

In neonates and infants, we routinely do a subtotal closure of the atrial level shunt to allow selective right-to-left shunting. In children, we leave a small residual atrial level shunt when there is moderate RV dysfunction or enlargement. However, in adults, the atrial level shunt is closed completely because of the small risk of paradoxical embolism in the older population.

The BDCPA is reserved for more significant degrees of RV systolic dysfunction or severe RV enlargement. When profound RV dysfunction (or RV enlargement) is present, we include the BDCPA as part of the complete repair (ie, cone repair, RV plication, reduction atrioplasty). When $\mathrm{RV}$ dysfunction is moderate to severely depressed, we perform the complete repair and separate from cardiopulmonary bypass, perform postbypass TEE analysis, and observe hemodynamics. If hemodynamics are not stable and there is an escalating inotrope requirement, then we resume bypass and proceed with BDCPA. In situations in which hemodynamics are marginal but appear to be stabilizing, we plan a delayed sternal closure in an effort to avoid the BDCPA. Persistent metabolic acidosis, low urine output, or rising creatinine reflect low cardiac output and the need for BDCPA becomes obvious.

\section{POSTBYPASS MANAGEMENT}

Separation from bypass is usually accomplished with low-dose epinephrine and milrinone infusion. Nitric oxide is used liberally when there is significant RV dysfunction. In general, we prefer faster heart rates of 100-120 bpm; atrial pacing is applied if the intrinsic heart rate is less than $100 \mathrm{bpm}$. In addition, we prefer relative hypovolemia with a CVP of $<10-12 \mathrm{~mm} \mathrm{Hg}$; the combination of mild hypovolemia and mild tachycardia keeps the RV smaller and reduces tension on the numerous sutures line of the reconstruction and minimizes excessive stretch of the RV. Postbypass TEE is performed with a physiologic systolic blood pressure (infants $80 \mathrm{~mm} \mathrm{Hg}$, children $100 \mathrm{~mm} \mathrm{Hg}$, and adults $120 \mathrm{~mm} \mathrm{Hg}$ ). Important elements of TEE analysis include: (1) degree of TR; (2) flow characteristics through the valve (laminar vs turbulent) and mean gradient across the proximal tricuspid orifice and the distal cone orifice; and (3) systolic function of the left and right ventricles with attention to regional wall abnormalities that would indicate a potential coronary problem (in the distribution of the right coronary artery or posterior descending). Finally, the presence of ventricular arrhythmias should be a clue to coronary artery compromise.

Volume administration is performed slowly to avoid acute RV distention. We maintain this relatively faster heart rate/hypovolemia strategy for the first 24 hours postoperatively, after which the blood pressure and heart rate are allowed to return normal levels. This strategy of hypovolemia and tachycardia may result in a mild metabolic acidosis that gradually resolves over the first 6 to 12 hours. Extubation from mechanical ventilation is still performed in the first 6-12 hours assuming the patient is warm, well perfused 
with normal hemodynamic parameters, and good urine output.

\section{Conflict of Interest Statement}

The author reported no conflicts of interest.

The Journal policy requires editors and reviewers to disclose conflicts of interest and to decline handling or reviewing manuscripts for which they may have a conflict of interest. The editors and reviewers of this article have no conflicts of interest.

\section{References}

1. da Silva JP, Baumgratz JF, da Fonseca L, Franchi SM, Lopes LM, Tavares GMP, et al. The cone reconstruction of the tricuspid valve in Ebstein's anomaly.
The operation: early and midterm results. J Thorac Cardiovasc Surg. 2007; 133:215-23.

2. Holst KA, Dearani JA, Said SM, Pike RB, Connolly HM, Cannon BC, et al. Improving results of surgery for Ebstein anomaly: where are we after 235 cone repairs? Ann Thorac Surg. 2018;105:160-9.

3. Dearani JA, Said SM, O'Leary PW, Burkhart HM, Barnes RD, Cetta F. Anatomic repair of Ebstein's malformation: lessons learned with reconstruction. Ann Thorac Surg. 2013;95:220-8.

4. Dearani JA, Said SM, Burkhart HM, Pike RB, O'Leary PW, Cetta F. Strategies for tricuspid re-repair in ABS done malformation using the cone technique. Ann Thorac Surg. 2013;96:202-10.

5. Leung MP, Baker EJ, Anderson RH, Zuberbuhler JR. Cineangiographic spectrum of Ebstein's malformation: its relevance to clinical presentation and outcome. J Am Coll Cardiol. 1988;11:154-6.

6. Saran N, Dearani JA. Tricuspid valve repair: how I teach it. Ann Thorac Surg. 2018;105:675-9.

Key Words: Ebstein anomaly, surgery, cone repair 